\title{
Information in Science and Buddhist Philosophy: Towards a non-Materialistic Worldview
}

\author{
Carlos Gershenson ${ }^{1}$
}

\begin{abstract}
Information theory has been developed for seventy years with technological applications that have transformed our societies. The increasing ability to store, transmit, and process information is having a revolutionary impact in most disciplines. The goal of this work is to compare the formal approach to information with Buddhist philosophy. Considering both approaches as compatible and complementary, I argue that information theory can improve our understanding of Buddhist philosophy and vice versa. The resulting synthesis leads to a worldview based on information that overcomes limitations of the currently dominating physics-based worldview.
\end{abstract}

Keywords: information theory, epistemology, Buddhism, worldviews, causality.

\section{Introduction}

One can identify three revolutions that have severely transformed human societies: Agricultural, Industrial, and Informational. We can say that the first one dealt with the control of matter, the second with the control of energy, and the third one,

1 Universidad Nacional Autónoma de México, Massachussetts Institute of Technology \& ITMO University. 
obviously, with the control of information. The exponentially increasing rate at which we can store, process, and transmit information is having an impact without precedents in all domains.

In science, computers have been tools with which we can explore complexity (Pagels, 1989). Our innate cognitive abilities limit how many variables we can consider in a mathematical model, but computers allow us to explore spaces of millions of variables. Complexity comes from the Latin plexus, which means intertwined (similar to the Sanskrit tantra). In other words, complex systems are difficult to separate, because elements have relevant interactions among them. This means that studying elements of a complex system in isolation is by definition incomplete, as interactions generate novel information that determines the future of elements and thus of the system. The recent study and understanding of complexity is having an impact in philosophy (Heylighen, Cilliers \& Gershenson, 2007; Gershenson, 2013), where the traditional scientific reductionistic worldview has shown to be obsolete. Information theory has been one of the basis for the development of digital computers, and the scientific, technological, and philosophical advances brought by these developments are providing a worldview that is compatible with Buddhist concepts. Also, advances in our understanding and formalization of information can be used to empirically test and explain Buddhist ideas.

In the next section, a general overview of information theory is given. Then this is applied to several perspectives of Vajrayana Buddhist philosophy. In Section 4, causality is discussed within this context. Finally, the implications of a 
worldview compatible with information theory and Buddhism are sketched in Section 5 before drawing Conclusions.

\section{Information Theory}

Information can be seen as a pattern or organization, as opposed to randomness. One can have the same matter, but depending on its arrangement, it will acquire different properties. For example, the same carbon atoms can be arranged as charcoal, diamonds, nanotubes, buckyballs, etc.: materials with drastically different properties. Kauffman (2000) used the "blender thought experiment" to illustrate the relevance of information: If you put all of our biosphere in a giant blender and switch it on, seconds later the molecular diversity of the planet would still be there, but soon it would decay, as the organization that maintains and produces complex molecules, i.e. life, would be lost. Traditional physics disregards information (meaning included), focussing only on objects and not on their organization. Still, from the examples above it is clear that the arrangement of matter is essential to describe phenomena at multiple scales, i.e. how components determine a system (upward causation) and how the system regulates components (downward causation).

Shannon (1948) formalized information, using an equivalent to BoltzmannGibbs entropy. He was interested on how messages could be reliably transmitted over a noisy channel. This can be measured in bits: binary variables which have been used in most information technologies. Bits are not zeroes or ones, but the potential of having zeroes or ones; i.e. one bit can be zero or one, two bits can 
carry zero (00), one (01), two (10), or three (11), three bits can be any number between zero and seven, $N$ bits can be any number between zero and $2^{N-1}$.

Shannon's information is minimal when the probability of one symbol (in the binary case, zero or one) is maximal, i.e. a message carries only zeroes or only ones. Thus, new bits do not carry information, as they can be predicted from the probability distribution. Previous patterns tell us the whole story. Shannon's information is minimal when the probability of symbols is homogeneous, i.e. there is the same probability of receiving zeroes or ones based on the already received bits, as in tossing a fair coin. No matter how many bits we have, all the information about the next bit will be carried by it. Previous patterns do not tell us anything. Fully known patterns carry no new information, while maximum information is carried by unknown patterns.

Variations of Shannon's information have led to the proposal of several other measures of information and entropies (Wehner \& Winter, 2010), some with roots in thermodynamics, quantum theory, or statistical mechanics. The technical details of information theory are omitted, since the arguments presented here do not depend on them and require an advanced level of mathematics. Still, interested readers are referred to Prokopenko, et al. (2009) and Fernández, et al. (2014).

Names are information, so anything we can name can be measured and described in terms of information theory. And those things we cannot name, we cannot speak about. So, by definition, information theory can be applied to everything (Gershenson, 2012). 


\section{Space}

In Buddhist traditions, and in particular in the Diamond Way (Skt. Vajrayana) (Nydahl, 2008), information is used like a metaphor: space is information. This can be interpreted as the ability of space of having an arbitrary number of patterns. In this sense, information is also a potential, as in information theory.

It is also said that space is bliss. If space has the potential of containing any pattern, then it is our decision which patterns to project in it. And a wise choice would be patterns that are joyful.

This idea of potential is furthermore related to the nature of mind: "the nature of mind is inherently like space; it includes everything you experience" (Thrangu, 2002). Mind is not clearly defined in Buddhist philosophy nor in cognitive science, but we could describe it as the perceiver/observer of information. However, this perceiver/observer can also be described in terms of information. The nature of mind will be further discussed towards the end of this section.

This understanding of space or mind as information is certainly subjective. But at the end, all epistemology has a certain subjectivity, as it is observers who describe phenomena and assign meaning to information. In this sense, even ontology is epistemological, because it is us observers who can speak about "being". Still, we can take a pragmatic approach like Wittgenstein (1999) and suggest that the meaning is given by the use information has. Shannon, in his original work, assumed meaning/decoding to be given, but it is clear that the same 
information (pattern) can acquire different meaning depending on the decoder (agent perceiving it).

In Buddhism, it is considered that suffering comes from ignorance (not knowing things as they are). One could say that particular types of ignorance occur when we consider objects as being real (materialism), when we consider subjects as being real (ego), or when we consider perception (action) to be real (mental veils or disturbing emotions). They cannot be real by themselves as they require the other two. Still, all three can be described in terms of information.

The Buddha truth state (Dharmakaya Skt., Chüku Tib.) can be interpreted as the realization of the inseparability of subject, object, and action (Varela \& Poerksen, 2006). A pattern (objective substrate) requires an observer (subject to give meaning) to decode information (action). But any observer also requires a substrate, i.e. subject is also an object. And without decoding, one cannot have any meaning in neither object nor subject.

Being aware of the inseparability of object, subject and action is still limited, as we perceive it as second-order observers. We can take a higher view and perceive ourselves perceiving, and this is still limited. We can continue this, perceiving how we perceive perceiving ourselves. We could say that illumination is reached when we manage to be $N^{\text {th-order }}$ observers and $N$ tends to infinity. With this highest view, there is no longer a sense of self, as everything is included: "awareness without center or limit, always and everywhere".

An observer can describe any object at different scales. Lower scales (more details) require more information, as more distinctions are considered. Higher scales require less information to describe them. The highest scale would require 
no information (when scale tends to infinity, information tends to zero), because there are no distinctions: everything is included (See Figure 1). In this sense, illumination would be a state with zero information. Also, the lowest scale implies infinite information, as all possible distinctions are included. Thus, everything is possible.

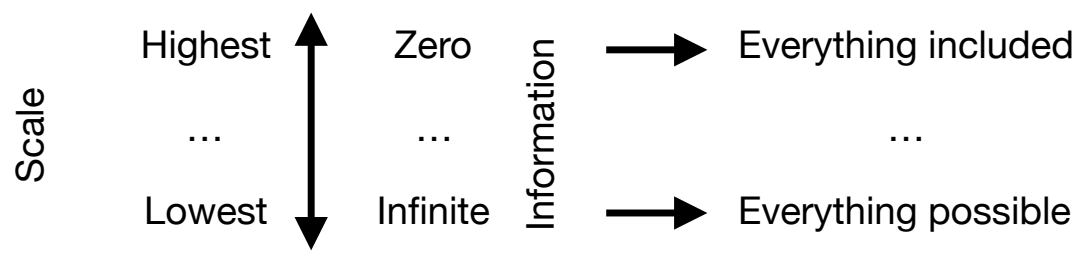

Figure 1. The relationship between scales of description and information.

Information can help clarify the concept of "innate coemergent wisdom" (Sahaya jñana $S k t$. Lhenchig kyepei yeshe Tib.), which states that mind is unborn. This can be said because information potential is always there. For example, the concept "four" is independent of its instantiations. We can have four apples, four pears, but "four" does not change. We can eat or harvest apples, so we won't have four apples anymore, but the concept "four" does not change. It was there before we had apples or pears. It was before we named it "four" and will always be there, as it is an arbitrary pattern. In a similar way, with unlimited space all possible patterns can be present, and the changes of the instances of a particular pattern do not change the potential of information, nor the potential of mind for perceiving them. This implies that it is useful not to identify with object (pattern) nor with subject (interpreter/meaning), as both will change, but with information itself. That has 
always been there and will always be. This can explain the idea of mind as potential, empty in essence, the basis for everything (Nydahl, 2011).

\section{Causality}

It is problematic to define reality. This leads to different considerations of what is real, depending on the context. For example, materialism considers only matter and energy to be real, everything else being epiphenomena. In different contexts, it could be argued that other phenomena are real, or that matter and energy are not real. To avoid these complications, one can use causality as a proxy for reality. If something has a causal effect on matter or energy, we can say that it is as (un)real as matter and energy.

One example can be seen with money. It is not purely material, as one cannot distinguish molecules of valuable bills from those which are worthless. Similarly, the digital bits encoding your savings somewhere in a server cannot be easily distinguished by themselves from bits which encode other information. The value is not in the substrate, but in the information they carry. And this information can have a causal effect on matter and energy. For instance, a skyscraper is composed by matter which was transformed with the use of money. The laws of physics were not broken, but only following them, the natural resources used to build the skyscraper would still be underground. Money was (partially) a causal agent that enabled the extraction and transformation of those resources.

Materialism inevitably leads to dualism, as traditional physics cannot describe information in general and cognition in particular (meaning-making living 
organisms). A traditional approach also is limited when attempting to describe the causality of "mind over matter", i.e. psychological control of physiology, despite ample evidence (see below), because "mind" is not considered as real.

Still, using information to describe phenomena one can simply consider downward causation (Campbell, 1974), which is traditionally rejected by reductionist approaches (Gallagher \& Appenzeller, 1999). According to reductionist materialism, reality lies at the lowest scale of phenomena: elementary particles. These are combined and cause appearances at higher scales: atoms, molecules, organisms, societies. But it defends that causality only "goes up". Downward causation - the effect of higher scales to lower scales - is dismissed. Still, as we saw with the money example, a social agreement can have a causal effect on matter and energy. One can say that free will works in a similar way: we can manipulate matter and energy (e.g. throw a rock) because information/ organization at the organism level enables the conditions for throwing a rock. But only the physical laws do not consider the organization of elementary particles at multiple scales, so free will cannot be described in terms of physics (and is thus dismissed as a convenient metaphor). But if we consider information as real, then downward causality is perfectly admissible and furthermore we avoid the inconveniences of dualism.

Causality can also be formalized, based on logic (Pearl, 2009). Information theory is mathematical, not physical. Causality itself is information, not a physical force. 


\section{Worldviews}

A worldview is a descriptive model of the world (Aerts, et al., 1994; Vidal, 2012). Worldviews are useful not only to explain our world, but also to give us a direction in our goals and actions. Our species has had a broad variety of worldviews. One can say that an evolutionary process should select worldviews that give survival advantages to people holding them. As knowledge is accumulated, some worldviews become obsolete and must be revised (e.g. flat Earth).

In spite of several limitations of a materialistic-based worldview (Gershenson, 2013), it still prevails among many people. Physics cannot comprehend the essence of all things. It describes matter and energy, but it is limited to describe e.g. patience or greed. All things have information, so we can see information as the essence of all things.

In fact, in many countries the most popular worldviews are religion-based rather than science-based. Worldviews are not selected because they are right or wrong, but people choose them because of what they have to offer is convenient for them. Also, there is a strong tradition bias, making it difficult to change worldviews. Some people would even interpret a change of worldview as a treason against their ancestors.

Just like Buddhist philosophy, information theory and current cognitive science are pointing towards a worldview not centered on objective phenomena (studied traditionally by physics), but centered on information, which can represent object, subject, and action within the same formalism. Physics (matter and energy) 
can be seen as a particular type of information (useful to describe only objects, not subjects or actions), so it actually includes matter and energy as particular cases.

An information-based worldview gives us choice (not absolute, as it depends on the circumstances) and considers free will as real as matter and energy. This leads to responsibility: situations are the consequences of our actions (karma), as opposed to a traditional worldview, which states that things are predetermined, events are a consequence the laws of physics, and free will is really only a convenient description, just an epiphenomenon.

An information-based worldview will not change beliefs of people on its own. Its advantages and benefits have to be explored further and promoted. Not because of philosophical reasons, but because of pragmatic reasons: conflicts and suffering would be reduced. This worldview will propagate only if it is convenient for people.

Changes of information can be due to changes in object (pattern), subject (meaning) or action (decoding). That is another reason for why one cannot separate them. In this sense, creativity can be the results of a new pattern, a new meaning, or a new decoding.

Information theory opens the possibility to study formally "esoteric" or "mystic" experiences, such as the placebo effect (Kong, et al., 2013), and more generally the causal effect of psychology on physiology (e.g. Lutz, et al, 2008). There are many studies showing that the effect of meditation is "real" (Murphy, et $a l ., 1997)$, i.e. it has a causal effect on our bodies. If we relate "Buddha qualities" as information potential, it is clear that all beings already have that potential (as 
they can perceive information). Buddhism is valuable because its methods are useful to recognize this potential.

Suffering, confusion, attachment and other disturbing emotions are real, but temporary. They are "only" information patterns, which can have causal effect on other information, including matter and energy. Buddhist teachings offer methods that change "negative" patterns, i.e. those that yield suffering, into "positive" patterns, described as joy or bliss, and also to keep that state (holding the highest view).

It is said that there is an inseparability between samsara (conditioned world) and nirvana (illumination). From a perspective of information processing, it could be said that the same patterns/information can be interpreted differently by different observers/encodings. Thus, there is always the potential of perceiving anything as perfect. Buddhist practice aims at realizing that potential.

If we have an information-based worldview as described above, we can better interpret that our true essence is "timeless awareness beyond center or limit, which playfully manifests all": information is everywhere, so we can identify information as the essence of all phenomena. The potential of information has no beginning and will never end.

Apart from what was exposed here, describing the world in terms of information enables us to explore general laws that apply to everything that we can perceive and describe (Gershenson, 2012).

\section{Conclusions}


Buddhist teachings are in line with recent scientific and philosophical developments related to information. This work attempted to relate information and Buddhism to complement each other.

Science is the best method we have to generate knowledge and understanding. Buddhism offers methods to experience our own mind. They can be complementary as they have different approaches but their goals can be aligned (Wallace, 2003). This synthesis can be summarized viewing information as potential and mind as information. This can be all encompassing and effortless (zero information because everything is already there).

It is said that mind is beyond concepts. Since all ideas presented here are conceptual, they are also limited, thus sketches of "the real thing". Still, they aim at pointing towards the goal of Buddhist practice: the recognition of the nature of mind. Nevertheless, they should be taken with caution, as they do not (and cannot) represent Buddhist teachings fully.

Historically, science fought with faith religions (namely Christianity), but left a void on spirituality. Buddhism is a spiritual practice compatible with science , as both are empiric. Given the current globalization trend and spreading of knowledge, it seems feasible that Buddhist concepts will be adopted more broadly in society and philosophy in the coming decades, filling the empty spiritual niche. The purpose of this would be to have an empirically-based and falsifiable approach to happiness and well-being.

\section{References}


- Aerts, D., Apostel, L., De Moor, B., Hellemans, S., Maex, E., Van Belle, H., and Van der Veken, J. (1994). World views. From Fragmentation to Integration. VUB Press, Brussels, Belgium.

- Campbell, D. T. (1974). 'Downward causation' in hierarchically organized biological systems. In Ayala, F. J. and Dobzhansky, T. (Eds.), Studies in the Philosophy of Biology, pages 179-186. Macmillan.

- Fernández, N., Maldonado, C., and Gershenson, C. (2014). Information measures of complexity, emergence, self-organization, homeostasis, and autopoiesis. In Prokopenko, M., editor, Guided Self-Organization: Inception, volume 9 of Emergence, Complexity and Computation, pages 19-51. Springer, Berlin Heidelberg.

- Gallagher, R. and Appenzeller, T. (1999). Beyond reductionism. Science, 284(5411):79-79.

- Gershenson, C. (2012). The world as evolving information. In Minai, A., Braha, D., and Bar-Yam, Y., editors, Unifying Themes in Complex Systems, volume VII, pages 100-115. Springer, Berlin Heidelberg.

- Gershenson, C. (2013). The implications of interactions for science and philosophy. Foundations of Science, 18(4):781-790.

- Heylighen, F., Cilliers, P., and Gershenson, C. (2007). Complexity and philosophy. In Bogg, J. and Geyer, R., editors, Complexity, Science and Society, pages 117-134. Radcliffe Publishing, Oxford.

- Kauffman, S. A. (2000). Investigations. Oxford University Press, Oxford, UK.

- Kong, J., Spaeth, R., Cook, A., Kirsch, I., Claggett, B., Vangel, M., Gollub, R. L., Smoller, J. W., and Kaptchuk, T. J. (2013). Are all placebo effects equal? 
placebo pills, sham acupuncture, cue conditioning and their association. PLOS ONE, 8(7):1-9.

- Lutz, A., Brefczynski-Lewis, J., Johnstone, T., and Davidson, R. J. (2008). Regulation of the neural circuitry of emotion by compassion meditation: Effects of meditative expertise. PLOS ONE, 3(3):1-10.

- Murphy, M., Donovan, S., and Taylor, E. (1997). The physical and psychological effects of meditation: A review of contemporary research. Institute of Noetic Sciences: Petaluma.

- Nydahl, O. (2008). The Way Things Are: A Living Approach to Buddhism for Today's World. O Books, London.

- Nydahl, O. (2011). The Great Seal: Limitless Space \& Joy: The Mahamudra View of Diamond Way Buddhism. Firewheel Publishing.

- Pagels, H. R. (1989). The Dreams of Reason: The Computer and the Rise of the Sciences of Complexity. Bantam Books.

- Pearl, J. (2009). Causality. Cambridge University Press.

- Prokopenko, M., Boschetti, F., and Ryan, A. (2009). An information-theoretic primer on complexity, self-organisation and emergence. Complexity, 15(1):11 28.

- Shannon, C. E. (1948). A mathematical theory of communication. Bell System Technical Journal, 27(3 and 4):379-423 and 623-656.

- Thrangu, R. (2002). The Life of Tilopa \& the Ganges Mahamudra. Namo Buddha Publications.

- Varela, F. J. and Poerksen, B. (2006). Truth is what works: Francisco J. Varela on cognitive science, Buddhism, the inseparability of subject and object, and 
the exaggerations of constructivism-A conversation. The Journal of Aesthetic Education, 40(1):35-53.

- Vidal, C. Metaphilosophical criteria for worldview comparison. Metaphilosophy, 43(3):306-347.

- Wehner, S. and Winter, A. (2010). Entropic uncertainty relations - a survey. New Journal of Physics, 12(2):025009.

- Wallace, B. A. (Ed.) (2003). Buddhism and science: Breaking new ground. Columbia University Press.

- Wittgenstein, L. (1999). Philosophical Investigations. Prentice Hall, 3rd edition. 